January 2005

\title{
Electroconvulsive Therapy: A Review of Its Current Status
}

Tyler Kenning

Thomas Jefferson University

Ranjnish Mago

Thomas Jefferson University

Steven Huege

Thomas Jefferson University

Kenneth Certa

Thomas Jefferson University

Rodney Pelchat

Thomas Jefferson University

Follow this and additional works at: https://jdc.jefferson.edu/jeffjpsychiatry

Part of the Psychiatry Commons

Let us know how access to this document benefits you

\section{Recommended Citation}

Kenning, Tyler; Mago, Ranjnish; Huege, Steven; Certa, Kenneth; and Pelchat, Rodney (2005)

"Electroconvulsive Therapy: A Review of Its Current Status," Jefferson Journal of Psychiatry. Vol. 19 : Iss.

1 , Article 1.

DOI: https://doi.org/10.29046/JJP.019.1.001

Available at: https://jdc.jefferson.edu/jeffjpsychiatry/vol19/iss1/1

This Article is brought to you for free and open access by the Jefferson Digital Commons. The Jefferson Digital Commons is a service of Thomas Jefferson University's Center for Teaching and Learning (CTL). The Commons is a showcase for Jefferson books and journals, peer-reviewed scholarly publications, unique historical collections from the University archives, and teaching tools. The Jefferson Digital Commons allows researchers and interested readers anywhere in the world to learn about and keep up to date with Jefferson scholarship. This article has been accepted for inclusion in Jefferson Journal of Psychiatry by an authorized administrator of the Jefferson Digital Commons. For more information, please contact: JeffersonDigitalCommons@jefferson.edu. 


\title{
Electroconvulsive Therapy: A Review of Its Current Status
}

\author{
Tyler Kenninga ${ }^{a}$, Rajnish Mago, M.D.b, Steven Huege, M.D.c, \\ Kenneth Certa, M.D.d, and Rodney Pelchat, M.D.e
}

\begin{abstract}
This article presents an overview of the current knowledge and practice of ECT. It provides a brief history of the development of ECT followed by a discussion of the accepted indications for ECT in psychiatric practice, including major depression, bipolar disorder (mania and depression), and schizophrenia. Moreover, a description of the technique of performing ECT is given. Also examined are the use of ECT in medically ill patients and the risks associated with ECT. In conclusion, with the aid of advances in anesthesia and the delivery of ECT, electroconvulsive therapy is a safe and effective treatment of mood and psychotic disorders that have been resistant to other treatment modalities.
\end{abstract}

Key Words: electroconvulsive therapy, ECT

\section{Introduction}

Despite being one of the most efficacious treatment modalities in all of psychiatry, and perhaps all of medicine, electroconvulsive therapy (ECT) remains surrounded by controversy. This stems largely from misconceptions and biases about ECT and is supported by widespread misinformation by the media and the internet. Because it uses electricity to induce a therapeutic seizure, ECT understandably appears frightening and hazardous to many laypeople. Further hindering the use of ECT are the numerous reports alleging permanent brain damage as a result of the therapy, a claim that has been disproved by repeated clinical trials, and by neuroimaging and autopsy studies. Regardless of such criticism, electroconvulsive therapy is a relatively safe and successful treatment modality for patients suffering from a number of Axis I illnesses. Separating itself from its outdated counterparts of insulin coma and frontal lobotomy, ECT continues to be utilized today due to a progressive refinement in the technique and its associated anesthesiological interventions. In fact, today, over 11,000 patients in Britain and roughly 50,000-100,000 patients in the United States receive ECT each year with an annual cost of approximately $\$ 200$ million $(1,2)$. 


\section{History}

In 1934, Hungarian psychiatrist Ladislas J. von Meduna observed that spontaneous seizures often had an anti-schizophrenic effect. Combining this observation with the then common, yet incorrect, belief that schizophrenia and epilepsy could not coexist in the same patient, von Meduna hypothesized that iatrogenically induced seizures in severe schizophrenics should have an effect similar to spontaneous seizures. Using intramuscular injections of camphor suspended in oil, he induced seizures in these patients to achieve successful remission of catatonia and other schizophrenic symptoms. Intravenously administered pentylenetetrazol eventually replaced oil of camphor in von Meduna's treatments and became an effective treatment for four years before the introduction of electrically-induced seizures in 1938 by Italian neuropsychiatrists Ugo Cerletti and Lucia Bini. These two administered the first electroconvulsive treatment in Rome. It was initially known as electroshock therapy (EST), but later became known as ECT. Psychiatrists from the University of Rome eventually brought the treatment to the United States and treatment began at The Psychiatric Institute in New York in 1940.

Two major problems existed with early ECT: 1) patients' discomfort caused by the procedure, and 2) bone fractures resulting from the motor activity of the seizure. These problems were eventually resolved by the use of general anesthetics and pharmacological muscle relaxation during treatment. An American psychiatrist, Abram E. Bennett, helped develop the method for extracting pure curare from plant material and later suggested the use of this substance along with spinal anesthetics during ECT. In 1951, succinylcholine was introduced and became the most widely used muscle relaxant for ECT and continues to be used today.

Until the 1950s and the introduction of the phenothiazines for psychiatric disorders, ECT was prominently used for the somatic treatment of major mental illness. With the increasing availability of neuroleptics, antidepressants, and mood stabilizers, ECT became progressively less utilized. Since the mid-1970s, however, refinement in the awareness of when and how to use ECT safely has given it an established and respected place alongside medications in the treatment of mental illness (3).

\section{Mechanism of Action}

ECT induces seizures in normal neurons by application, through the scalp, of a carefully-controlled current. This electrical stimulus can be regulated to produce a seizure of a necessary duration to incur the most therapeutic benefit. Due to the high impedance of the skin's oily secretions and the skull, however, only about twenty percent of the applied charge actually enters the skull to excite neurons (4).

Induction of a bilateral generalized seizure is necessary for ECT. In studies using EEG to study ECT, it has been shown that after this generalized seizure a period of about 60-90 seconds of postictal suppression occurs. There is a return to pre-seizure appearances in about thirty minutes. An interesting finding is that seizures cause an increase in cerebral blood flow, the use of glucose and oxygen, and the permeability of the blood-brain barrier. After the seizure, blood flow and glucose metabolism decrease, most markedly in the frontal lobes, and this decrease appears to correlate strongly with the therapeutic outcome of ECT. Therefore, the effects on frontal lobe function, an area strongly implicated in the pathophysiology of depressive illness, may be relevant in the mechanism of action of ECT $(5,4,6)$.

Further research has focused on changes in neurotransmitters, neuroreceptors and second-messenger systems. Virtually every neurotransmitter system is affected by ECT, but consistent findings include downregulation and desensitization of postsynaptic beta-adrenergic receptors and upregulation of postsynaptic serotonin receptors, a change similar to that observed with virtually all antidepressant treatments ECT also decreases serotonin turnover and acetylcholine levels in the brain and CSF and also may result in reduction in dopamine levels. Regarding second-messenger systems, ECT affects the coupling of G proteins to their receptors, the activity of adenylyl cyclase and phospholipase $\mathrm{C}$, and the regulation of calcium entry into neurons $(7,8,4)$. 


\section{Indications}

The three clearest indications for ECT are major depressive disorder, manic episodes, and schizophrenia.

\section{Indications for ECT}

- Major depressive episode (unipolar or bipolar)

- Mania, especially in the context of first-trimester pregnancy or history of neuroleptic malignant syndrome

- Schizophrenia, especially with floridly positive symptoms

- Miscellaneous conditions

- Parkinson's disease

- Neuroleptic malignant syndrome

\section{Major Depressive Episode (MDE):}

MDD is the most common indication for ECT with over eighty percent of ECT patients in the United States being treated for this. The response rate in such patients is $80-90 \%$, a number greater than or equal to antidepressant medications. ECT is effective for both unipolar as well as bipolar depression and should be considered as a treatment for patients who have failed medication trials, have not tolerated medications, have severe or psychotic symptoms, are acutely suicidal or homicidal, or have marked symptoms of agitation or stupor $(9,10)$.

Regarding specific variants of depressive disorder:

- Several studies have reported that delusional or psychotic depression is particularly responsive to ECT while others have found no difference in the response rates for ECT in psychotic and non-psychotic depressive disorders. Despite this, ECT should continue to be considered as the initial treatment for patients suffering from major depressive episodes with psychotic features, because this group of illnesses tends to be poorly responsive to antidepressant pharmacotherapy alone.

- MDD with melancholic features (markedly severe symptoms, psychomotor retardation, early morning awakening, diurnal variation, decreased appetite and weight, and agitation) may respond favorably to ECT.

- Depressed patients who are less likely to respond to ECT include those with somatization disorder (5)

- Despite a higher level of physical illness and cognitive impairment, even the oldest patients with severe major depression tolerate ECT in a manner similar to that of younger patients and demonstrate a similar or better acute response. Elderly patients tend to respond more slowly than younger patients, but age has been shown to positively influence response to treatment. In some studies, older age conferred a greater likelihood of achieving remission $(8,11,9)$. ECT should be considered in severely depressed and suicidal children/adolescents who may be less likely to respond to antidepressants than adults (3).

It should be noted that ECT does not provide prophylaxis against MDD unless it is administered on a longterm maintenance basis. It is generally recommended that a course of ECT be followed by some form of maintenance therapy. This is usually in the form of medication but maintenance ECT is an alternative ( 9 , 12). Finally, despite its efficacy as a treatment for major depression, no direct evidence exists demonstrating that ECT prevents suicide.

\section{Manic episodes:}

ECT is an efficacious treatment for manic and mixed episodes of bipolar disorder. ECT is at least equal to lithium in the treatment of acute manic episodes. Due to the tremendous efficacy of the pharmacological treatment and prophylaxis of manic episodes, however, the use of ECT is generally limited to situations with specific contraindications to these measures. ECT seems to be particularly indicated in the treatment of manic episodes if either the patient is in the first trimester of pregnancy (because of concerns about the ter- 
atogenicity of mood stabilizers) or has a history of neuroleptic malignant syndrome. Finally, it is important to remember that use of lithium along with ECT is believed to increase risk of delirium or other organic syndromes (13).

\section{Schizophrenia}

ECT is an effective treatment for symptoms of acute schizophrenia but not for those of chronic disease. Prominent positive symptoms, catatonia, or affective symptoms are most responsive to ECT. For these patients, ECT is as effective as antipsychotics but may produce a therapeutic response sooner. In addition, ECT is considered an efficacious treatment for related psychotic disorders, notably schizophreniform disorder and schizoaffective disorder (14).

\section{Other Potential Indications}

For other diagnoses, the efficacy data for ECT is only suggestive, or only a partial consensus exists in the field supporting its use. In such cases, ECT should be recommended only after standard treatment alternatives have been considered as a primary intervention.

- Episodic or atypical psychoses

- Obsessive-compulsive disorder

- Medical conditions: neuroleptic malignant syndrome, hypopituitarism, intractable seizure disorders, and the "on-off phenomenon" of Parkinson's disease

For each of the above indications, ECT may be the treatment of choice in those who are unable to take medication safely, including pregnant women, the elderly, and those with concomitant medical illnesses.

\section{ECT in Adolescents:}

Current medical recommendations for ECT in adolescents are basically similar to those for the adult population. In generally, ECT can be used when more conservative treatments have been unsuccessful. ECT may be considered when there is a lack of response to two or more trials of pharmacotherapy or when the severity of symptoms precludes waiting for a response to pharmacological treatment.

In one study of adolescents, mood disorders had a high rate of response to ECT (75-100\%), whereas psychotic disorders had a lower response rate (50-60\%). ECT was shown to be equally effective for adolescents and adults (58\% in each group achieved remission). The main difference was the diagnosis for which patients were referred: most of the adolescents suffered from psychotic symptoms whereas most of the adult patients had mood disorders (15).

\section{Contraindications}

Rather than being absolute, contraindications to ECT are better conceptualized as situations in which the patient is at increased risk of variable magnitude and has an increased need for close monitoring:

- Pregnancy: fetal monitoring is generally thought to be unnecessary unless the pregnancy is high risk or complicated

- Space-occupying CNS lesions with increased intracranial pressure (ICP): at increased risk for edema and brain herniation after ECT because of the exacerbation of ICP with ECT

- If the lesion is small, the risk of serious complications can be minimized with dexamethasone pretreatment, use of mannitol, and careful control of the patient's hypertension.

- Increased intracranial pressure or risk for cerebral bleeding (e.g., cerebrovascular diseases and aneurysms): at risk during ECT because the procedure acutely increases cerebral blood flow, mainly during the seizure

- Risk can be reduced, although not completely eliminated, with control of the patient's blood pressure during treatment 
- Pre-existing seizure disorders:

- Destabilization of seizure disorder or precipitation of status epilepticus

- ECT may make an epileptic patient temporarily more refractory to spontaneous seizures

- Anticonvulsants do not make ECT impossible, but may require the administration of a greater electrical stimulus. If the elicitation of adequate seizures is difficult in patients taking anticonvulsants for seizure disorder, it may be necessary to consider tapering the anticonvulsant dose in consultation with the patient's neurologist.

- If the patient is taking anticonvulsants purely for their mood-stabilizing qualities, then the decision to discontinue these medications is much easier

- Before proceeding with ECT, tapering or even discontinuing drugs such as benzodiazepines that are not used for anticonvulsant purposes but raise the seizure threshold should be considered.

- Recent myocardial infarction: These patients are another high-risk group, although the risk has been found to be greatly diminished 3-4 weeks post-event.

- ECT induces increases in pulse and blood pressure that result in a $200-300 \%$ increase in myocardial oxygen consumption during and for a few minutes after the seizure

- It is important to obtain optimal stabilization of cardiac status before treatment; a cardiology consult and pretreatment cardiac imaging may be required.

- Another strategy is the intravenous administration of beta-blockers immediately pretreatment to dampen the increase in myocardial oxygen consumption. If pretreatment beta-blockade is used, then an anticholinergic agent, such as glycopyrrolate or atropine, should also be given to minimize the risk of vagally-mediated prolonged aystole or bradycardia

- Pacemakers: usually do not need any special adjustment or attention aside from ensuring that monitoring and other equipment are well grounded. Patients with pacemakers and implantable cardioverter defibrillators (ICDs) usually can be safely treated with ECT $(16,17)$, but pretreatment consultation with a cardiac electrophysiologist is recommended. A physician experience in pacemaker and ICD management should be present at least during the first few ECTs to monitor the device and reset it if needed.

- Hypertension: Patients should be stabilized on their antihypertensive medications before ECT is administered. Beta blockers and sublingual nitroglycerin can also be used to protect such patients (3, $11,8,10)$.

- Severe aortic stenosis

\section{Concomitant Medications}

Concomitant medications must be assessed for effects on seizure threshold and for drug interactions with medications used during ECT.

- Antidepressants generally lower the seizure threshold, which may increase the duration of seizures or the probability of status epilepticus and spontaneous seizures. Certain antidepressants, such as tricyclics, may increase cardiac irritability when combined with ECT.

- Benzodiazepines and anticonvulsant mood-stabilizers, such as carbamazepine and valproic acid, make the elicitation of seizures more difficult. Because benzodiazepines have independent amnestic effects, there is concern, especially in elderly patients, that negative synergism with ECT may accentuate cognitive side effects.

- Anticonvulsant medications increase seizure threshold and interfere with seizure expression. When prescribed for a psychiatric indication, it is advisable to taper and discontinue them as rapidly as possible before an initial ECT course. When used for treatment of a seizure disorder, however, the morning dose is usually withheld before ECT.

- Lithium should be held because it can result in increased incidence of delirium and other organic syndromes when combined with ECT (13). 
- Buproprion should not be taken while undergoing a course of ECT, because it can produce lateappearing seizures.

- While there is a concern that clozapine may have undesirable effects on ECT, the combination has been tried with success in clozapine-refractory patients (18).

- Lidocaine should be held during ECT because it decreases the duration of both motor and EEG seizure activity (19).

- Theophylline, even with blood levels in the therapeutic range for asthma control, increases the duration of seizures and has been linked to status epilepticus during ECT. It should be discontinued whenever possible or its dosage should be kept as low as possible

\section{Technique of Electroconvulsive Therapy}

\section{Premedications}

Although not universally used anymore, muscarinic anticholinergic medications are administered before ECT to minimize oral and tracheobronchial secretions and to prevent bradycardia and asystole. The agents do remain indicated for patients on beta-blockers and for those with ventricular ectopic beats. Atropine is the drug of choice for attenuating or blocking ECT's direct vagal effects on the heart. Glycopyrrolate may be more favorable in certain patients as it is less likely to cross the blood-brain barrier and exacerbate postanesthesia confusion than is atropine. Of the two medications, however, atropine is more effective in preventing bradycardia and ectopy (20).

\section{Anesthetics}

ECT requires general anesthesia and oxygenation. Etomidate or barbiturates are used as anesthesia for ECT. It is also important for the depth of anesthesia to be as light as possible, not only to minimize adverse effects but also to avoid negatively influencing the seizure threshold, a characteristic associated with many anesthetics. It may be preferable in some patients to use etomidate for anesthesia as it does not increase the seizure threshold. This is especially helpful in elderly patients where seizures become more difficult to induce (7).

Muscle relaxants were added to the administration of ECT in order to eliminate the early problem of bone fractures and other injuries that resulted from the motor activity of the seizure. Following the onset of the anesthetic effect, a muscle relaxant is administered to ensure profound muscular relaxation without complete paralysis. In some patients at increased risk for injury, however, paralysis may be required. Such predisposing conditions include osteoporosis, spinal injury, or pacemakers. Succinylcholine, an ultrafast-acting depolarizing agent, has become the agent of choice for muscle relaxation in ECT $(7,20)$.

In the elderly, beta-adrenergic receptor antagonists like esmolol and labetalol frequently are used at times to minimize acute changes in heart rate or blood pressure.

\section{Electrode Placement}

ECT can be conducted with either bilaterally- or unilaterally-placed electrodes. Bilateral placement is arguably more efficacious than unilateral as patients tend to respond more quickly and fully with the former. Despite its more rapid therapeutic response, bilateral ECT produces more acute amnesia and greater confusion in the initial weeks after treatment. This effect is negligible, however, after two months as any difference between the two different lead placements is no longer apparent.

In bilateral placement, both electrodes are placed fronto-temporally, one over each hemisphere. With the unilateral technique, there are a number of varying placements, but the most commonly used has one electrode placed midline and the other placed fronto-temporally over the nondominant hemisphere, most often the right. 
Generally, treatment is initiated with unilateral placement because of its more favorable side effect profile and because, in countries other than the United States, of controversy over bilateral efficacy. If, after four to six treatments, a therapeutic response is not seen, then a switch to bilateral placement can be made. Bilateral ECT may be indicated initially in patients with severe symptoms or those who pose an immediate danger to themselves or others. Examples of such situations include catatonic stupor, marked agitation, acute suicide risk, severe depressive or manic symptoms, and treatment-resistant schizophrenia $(5,15,8,4)$.

\section{Stimulus}

The strength of the stimulus must be sufficient to induce a generalized seizure, but there is a great deal of variation, up to 40-fold, between individuals and their seizure thresholds. In addition, a course of ECT treatments often increases a patient's seizure threshold by $25-200 \%$. Adding to the confusion of inter- and intrapatient differences in seizure threshold, a difference in genders, with men having a higher threshold, and age, in which the elderly require a greater stimulus, has been established $(3,8,4)$.

Since determination of an individual seizure threshold is obviously not straightforward, it is often necessary to initiate ECT with an electrical stimulus that is sub-threshold and then increase the intensity by $100 \%$ for unilateral placement and by $50 \%$ for bilateral placement until the necessary threshold, and subsequent seizure, is achieved. Research indicates that stimuli up to 3-5 times threshold may have the quickest and greatest therapeutic response, while minimally suprathreshold doses cause the fewest and least severe cognitive adverse effects $(11,10)$.

\section{Seizure Duration}

Since the seizure is the therapeutic agent in ECT, an objective measure for determining the occurrence and even the duration of the induced seizure would be extremely helpful. Before the use of succinylcholine, there was never any question as to whether a seizure had occurred. Now that the tonic-clonic movements previously seen with ECT administration have been minimized with the use of muscle relaxants, there must be an alternative verification of seizure activity. With the application of the electrical stimulus, there is a simultaneous contraction of facial muscles, but this activity occurs regardless of whether a seizure does. Therefore, this observation alone cannot be relied on to ensure that a seizure has been induced. Instead, a technique known as the cuff method is generally used. This involves observing the convulsive movements in an extremity with a blood pressure cuff (either on the forearm or the calf) inflated well above systolic pressure before the succinylcholine is injected. Thus, the muscles distal to the cuff are isolated from the paralyzing effects and the visually monitored convulsion in the extremity provides a generally accurate measure of seizure occurrence and length. For a seizure to be of therapeutic value in ECT, it should have duration of at least twenty-five seconds $(15,7,11,10)$.

If a seizure of therapeutic value is not observed at a particular stimulus, readministration of stimulus can be attempted before other measures. In addition, it is important to realize that there may be some delay between the stimulus administration and the onset of seizure activity. If a particular stimulus is determined to be insufficient, first, the contact between the electrodes and the skin should be checked and then the stimulus can be increased $25-100 \%$. In addition, the anesthetic agent can be changed to minimize increases in seizure threshold. Other measures that lower the seizure threshold and will aid in the inducing of a seizure include hyperventilation and administration of parenteral caffeine $250-1000 \mathrm{mg}$ five to ten minutes before the stimulus $(21,15,7)$.

Seizures lasting greater than three minutes can result in status epilepticus and should be terminated with either additional doses of the barbiturate anesthetic agent or with IV diazepam. In addition, the patient should be intubated to ensure airway protection. Prolonged seizures are clinically significant, because they are associated with greater postictal confusion and amnesia and inadequate oxygenation resulting in increased hypoxia-related risks (cerebral and cardiovascular complications). 


\section{Frequency and Number}

The frequency and number of ECT treatments is determined by clinical response. Over the course of all clinical illness, the average number of treatments is five to ten during the initial course and these are generally administered three times per week. ECT can also be performed twice weekly as this schedule is associated with less memory impairment. Commonly, the treatment course of major depression can take six to twelve treatments (although up to twenty treatments may be required); treatment of manic episodes eight to twenty treatments; schizophrenia can take greater than fifteen treatments; catatonia and delirium may require as few as one to four. Treatment should continue, however, until the patient no longer continues to improve, having achieved the maximum therapeutic response. Further treatment will only result in greater adverse effects $(3,5)$.

Multiple monitored ECT involves the administration of multiple stimuli and the production of multiple therapeutic seizures during a single session. Usually, two bilateral stimuli are given in two minutes. The aim is to accelerate the treatment course and to reduce the required number of anesthesia inductions for a course of treatments. This may be helpful in severely ill patients or in those at increased risk for the adverse effects of anesthesia. Despite this benefit, multiple monitored ECT is associated with the greatest incidence of serious cognitive impairment (3) and the procedure is rarely used.

\section{Outpatient Treatment}

Except in a few select cases, ECT can be performed safely and effectively on an outpatient basis. In fact, out patient ECT may be preferable to inpatient as the patient is permitted to remain in his or her home environment and the overall costs of the procedure are lessened. The main factor that would preclude outpatient treatment would be the severity of a patient's psychiatric condition. If outpatient treatment is to be undertaken, the patient should still be carefully observed during the treatment and post-treatment recovery phases as these are the periods that pose the greatest physical risk to the patient $(3,22,2,11,1)$.

\section{Maintenance Treatment}

Although ECT can be rapidly effective, a course of treatments does not usually result in a long-term remission of symptoms. Instead, as noted above, maintenance therapy, usually in the form of pharmacotherapy, should always be considered. ECT itself, however, can also be used for maintenance therapy on a variety of schedules (weekly, biweekly, monthly) and has been reported to be effective in preventing relapse. Maintenance ECT may be indicated in patients who suffered a rapid relapse after their initial ECT treatment, who have severe or psychotic symptoms, or who are unable to tolerate pharmacotherapy.

Finally, if a course of ECT treatments does not produce a therapeutic response, there are anecdotal reports that previously attempted pharmacotherapy, even if ineffective originally, can be retried and may result in improvement $(2,9,12)$.

\section{Adverse Effects}

\section{Mortality}

The mortality rate for ECT is extremely low, further supporting the safety claim of the treatment. Rates of about $0.002 \%$ per treatment and $0.01 \%$ for each patient have been reported. As a reference, the per-treatment mortality rate compares very favorably with the mortality rate of childbirth, $0.01 \%$, in the United States (3). Death from ECT, when it does occur, is usually from cardiovascular complications and is most likely to occur in patients whose cardiac status is already compromised. 


\section{CNS Effects}

Headache (up to $45 \%$ ), confusion, and even delirium shortly after the seizure while the patient is coming out of anesthesia are common. Marked confusion may occur in up to $10 \%$ of patients within thirty minutes of the seizure. Delirium is usually most pronounced after the first few treatments, in patients who receive bilateral ECT, or who have pre-existing neurological disorders. The confusion characteristically clears within days or a few weeks at the longest.

Despite numerous claims otherwise, there has been no evidence of structural brain damage caused by ECT. Repeated studies have supported this fact using several brain-imaging modalities. Also, in animal electroconvulsive shock studies using parameters analogous to those used in ECT, histopathologic studies reveal no structural neuronal damage. Finally, autopsies of patients who received ECT do not suggest a pattern of CNS damage caused by ECT.

It is more difficult, however, to rule out permanent functional CNS damage resulting from ECT. Some patients do note cognitive changes persisting for extended periods with the greatest being the association between ECT and memory loss. Approximately seventy-five percent of ECT patients say that memory impairment is the worst adverse effect. Studies based on neuropsychological testing of ECT patients show that anterograde amnesia (the ability to form new memories) can persist for at least 3 months after a course of ECT. These patients have difficulty laying down permanent memories of some events that take place just before, during, and immediately after a course of ECT. Memory impairment after a course of ECT is extremely common. However, follow-up data indicate that almost all patients are back to their cognitive baseline after six months. Some patients, however, complain of persistent memory difficulties. The degree of cognitive impairment during treatment and the time it takes to return to baseline appear to be related in part to the amount of electrical stimulation used during treatment. Also, memory impairment is most often reported by patients who have experienced little improvement with ECT. This may be due to the escalating intensity of stimulation given to these patients before ECT is abandoned.

Most studies evaluating the potential effects of ECT on memory loss have used neuropsychological testing to evaluate the ability to form new memories. These studies have shown that the ability to form new memories is not significantly impaired by ECT. However, the there is less data on patients' loss of pre-existing or autobiographical memories. As many patients complain of retrograde as well as anterograde memory loss, more investigation of this potential side-effect is necessary (1).

Tardive seizures, those occurring some time after the treatment course, may develop in patients with a preexisting seizure disorders, and in some rare patients, ECT can even precipitate the development of a seizure disorder. These conditions should be managed clinically as if they were pure epileptic disorders $(3,5,15$, $23,7)$.

Occasionally, mild transient cardiac arrhythmias may occur during ECT, particularly in patients with a preexisting cardiac disease. These arrhythmias are usually a by-product of brief postictal bradycardia and, therefore, can often be prevented by increasing the dosage of an anticholinergic premedication. Other arrhythmias are secondary to the tachycardia during the seizure and may occur as the patient returns to consciousness. A prophylactic beta-blocker can be useful in such cases.

\section{Additional concerns:}

- An apneic state may be prolonged if metabolism of succinylcholine is impaired

- Sore muscles resulting from seizure motor activity can generally be alleviated by pretreatment with curare or atracurium or by increasing the dose of succinylcholine.

- Minor adverse effects, including nausea, vomiting, muscle aches, confusion and agitation, rarely per- 
sist beyond the day of treatment. Some of these are secondary to the anesthetic agent and some are secondary to the ECT treatment itself.

In balancing ECT's effectiveness versus its risks, it can be concluded that forms of ECT that produce better treatment outcomes (e.g, bilateral, high dose) are more often associated with greater side-effects, especially cognitive ones. Thus, the physician and patient must together balance the relative value and urgency of symptom relief versus the imperative to avoid cognitive side-effects (24).

\section{Conclusions}

Despite continuous advances in psychopharmacology, psychiatric disorders are often refractory to medications. ECT has been administered worldwide for seventy years, even in patients with significant concurrent medical or neurologic illness. However, despite its proven safety and efficacy, ECT does have disadvantages including the requirement of general anesthesia and the production of cognitive adverse effects. Two new, emerging techniques, transcranial magnetic stimulation and vagus nerve stimulation, may eventually at least partially satisfy the need for treatment methods that will provide the same clinical benefits as ECT but with fewer adverse effects. However, at this time, electroconvulsive therapy remains a highly potent modality for the treatment of severe and refractory axis I disorders. However, education of patients, families, and even medical professionals is essential for acceptance of ECT. A book we recommend for this purpose is Electroshock: Restoring the Mind (25).

\section{REFERENCES}

1. Rose D, Fleischmann P, Wykes T, Leese M, Bindman J. Patients' perspective on electroconvulsive therapy: systematic review. British Medical Journal 2003; 326 (7403): 1363.

2. Espinoza R. Electroconvulsive therapy in the long-term care setting: an overview of controversies in practice. Journal of the American Medical Directors Association 2004; 5(2): S54-8.

3. Abrams R. Electroconvulsive Therapy. Oxford: Oxford University Press, 4th Edition, 2002.

4. Kotresh S, Girish K, Janakiramaiah N, Rao GU, Gangadhar BN. Effect of ECT stimulus parameters on seizure physiology and outcome. Journal of ECT 2004; 20(1): 10-12.

5. Benbadis SR, Tatum WO. Advances in the treatment of epilepsy. American Family Physician 2001; 64 (1): 91-98.

6. Rasmussen KG, Sampson SM, Rummans TA. Electroconvulsive therapy and newer modalities for the treatment of medication-refractory mental illness. Mayo Clin Proc 2002; 77 (6): 552-6.

7. Ding Z, White PF. Anesthesia for electroconvulsive therapy. Anesthesia \& Analgesia 2002; 94 (5): 1351 64.

8. Kamat SM, Lefevre PJ, Grossberg GT. Electroconvulsive therapy in the elderly. Clinics in Geriatric Medicine 2003; 19 (4): 825.

9. Pagnin, D et al. Efficacy of ECT in depression: a meta-analytic review. Journal of ECT 2004; 20 (1): 13-20. 
10. Rasmusssen, KG. Electroconvulsive therapy in the medically ill. Psychiatric Clinics of North America 2002; 25 (1): 177-93.

11. Kelly KG, Zisselman M. Update on electroconvulsive therapy (ECT) in older adults. Journal of the American Geriatrics Society 2000; 48 (5): 560-6.

12. Sackeim HA, Haskett RF, Mulsant BH, Thase ME, Mann JJ, Pettinati HM, Greenberg RM, Crowe RR, Cooper TB, Prudic J. Continuation pharmacotherapy in the prevention of relapse following electroconvulsive therapy: a randomized controlled trial. JAMA 2001; 285 (10): 1299-1307.

13. Naguib M, Koorn R. Interactions between psychotropics, anaesthetics and electroconvulsive therapy: implications for drug choice and patient management. CNS Drugs 2002; 16(4): 229-47.

14. Etheridge K, Yarrow L, Peet M. Pathways to care in first episode psychosis. Journal of Psychiatric \& Mental Health Nursing 2004; 11(2): 125-8.

15. Bloch Y. Electroconvulsive therapy in adolescents: similarities to and differences from adults. Journal of the American Academy of Child and Adolescent Psychiatry 2001; 40 (11): 1332-6.

16. Dolenc TJ, Barnes RD, Hayes DL, Rasmussen KG. Electroconvulsive therapy in patients with cardiac pacemakers and implantable cardioverter defibrillators. Pacing Clin Electrophysiol 2004; 27(9):125763.

17. Giltay EJ, Kho KH, Keijzer LT, Leijenaar M, van Schaick HW, Blansjaar BA. Electroconvulsive therapy $(\mathrm{ECT})$ in a patient with a dual-chamber sensing, VDDR pacemaker. JECT 2005; 21(1): 35-8.

18. Kho KH, Blansjaar BA, de Vries S, Babuskova D, Zwinderman AH, Linszen DH. Electroconvulsive therapy for the treatment of clozapine nonresponders suffering from schizophrenia - an open label study. Eur Arch Psychiatry Clin Neurosci 2004; 254(6): 372-9.

19. Fu W, Stool LA, White PF, Husain MM. Acute hemodynamic responses to electroconvulsive therapy are not related to the duration of seizure activity. J Clin Anesth 1997; 9(8): 653-7.

20. Folk JW, Kellner CH, Beale MD, Conroy JM, Duc TA. Anesthesia for electroconvulsive therapy: a review. Journal of ECT 2000; 16 (2): 157-170.

21. Calev A, Fink M, Petrides G, Francis A, Fochtmann L. Caffeine pretreatment enhances clinical efficacy and reduces cognitive effects of electroconvulsive therapy. Convuls Ther 1993; 9(2): 95-100.

22. Dew R, Vaughn W. Efficiency of outpatient ECT. Journal of ECT 2004; 20(1): 24-5.

23. Dean CE, Borchardt CM. Nonconvulsive status epilepticus after electroconvulsive therapy: an additional case and risk factor. Journal of ECT 2004; 20(1): 54-5.

24. UK ECT Review Group. Efficacy and safety of electroconvulsive therapy in depressive disorders: a systematic review and meta-analysis. Lancet. 2003 Mar 8; 361(9360): 799-808.

25. Fink M. Electroshock: Restoring the Mind. Oxford University Press, Oxford. 1999. 